\section{Jerk Limited Time Optimal Control of Flexible Structures}

\section{Marco Muenchhof}

\section{Tarunraj Singh}

e-mail: tsingh@eng.buffalo.edu

Department of Mechanical and Aerospace Engineering, SUNY at Buffalo, Buffalo, NY 14260

This paper addresses the problem of designing jerk limited timeoptimal control profiles for rest-to-rest maneuvers of flexible structures. The variation of the structure of the jerk profile as a function of the permissible jerk is studied. An optimal control problem is formulated which includes constraints to cancel the poles corresponding to the rigid body and flexible modes of the system and to satisfy the boundary conditions of the rest-to-rest maneuver. The proposed technique is illustrated on the benchmark Floating Oscillator problem where the jerk profile is parameterized as a bang-off-bang or bang-bang profile.

[DOI: 10.1115/1.1543552]

\section{Introduction}

Vibration control of slewing flexible structures has been studied by numerous researchers with a variety of applications including maneuvering of large space structures [1], flexible arm robots [2], computer disk drives [3], and cranes [4]. In applications such as disk drives and cranes, the objective of the controllers is to minimize the maneuver time with quiescent final states. Additional constraints such as limits on fuel consumed, robustness to modeling uncertainties, maximum deformation permitted etc., have been included into the problem formulation. Motivated by Smith's Posicast Control [5], Singer and Seering [6] proposed a technique to design an input shaper addressing the issue of sensitivity of the Posicast controllers to uncertainties in damping and natural frequencies of the underdamped modes. Singh and Vadali [7] illustrated that a time-delay prefilter, which cancels the underdamped poles of the system, results in the same control profile as the input shaped controller. They also illustrated that the use of a series of time-delay filters results in increased robustness to modeling errors.

In this paper, the problem of designing time-optimal control profiles with constraints on the maximum magnitude of jerk is addressed. The proposed technique is illustrated on the benchmark two-mass/spring system undergoing a rest-to-rest maneuver. To account for the constraint on the jerk, a new state is introduced into the system model which represents the control input. The time rate of change of the control input, i.e., the jerk, will now represent the control input of this augmented system. Following the formulation of the optimization problem, the technique for the parameterization of the controller is discussed. It will be shown that the optimal jerk profile is bang-bang or bang-off-bang and varies with the magnitude of the maximum permitted jerk. The spectrum of control profiles includes regions where the constraints on the control are active and others where the constraints are inactive. In the inactive regions, the time-optimal control design requires the jerk profile to be bang-bang and techniques proposed

Contributed by the Dynamic Systems and Control Division of THE AMERICAN SOCIETY OF MECHANICAL ENGINEERS for publication in the ASME JOURNAL OF DYNAMIC SYSTEMS, MEASUREMENT, AND CONTROL. Manuscript received by the ASME Dynamic Systems and Control Division, April 2001; final revision, September 2002. Associate Editor: C. Rahn. in the literature $[8,9]$ can be used to verify optimality of the control profile. Numerical results are presented to illustrate the performance of the proposed controller.

\section{Problem Formulation}

This paper deals with the design of controllers for flexible structures, which can be described by the set of differential equations

$$
M \ddot{y}(t)+K y(t)=D u(t)
$$

where $M$ and $K$ are the mass and stiffness matrices. Representing $u(t)$ as a new state and $v(t)$ as the new input, the additional state equation is

$$
\dot{u}(t)=v(t)
$$

The optimal control problem can be stated as the design of the jerk profile which minimizes the cost function

$$
F=\frac{1}{2} T_{\text {final }}^{2}
$$

subject to the constraints

$$
-J \leqslant v(t) \leqslant J \forall t \in\left[0, T_{\text {final }}\right]
$$

and

$$
-1 \leqslant u(t) \leqslant 1 \forall t \in\left[0, T_{\text {final }}\right]
$$

where $J$ is the magnitude of permissible jerk and $T_{\text {final }}$ is the maneuver time. The boundary conditions for a rest-to-rest maneuver of unit displacement are given as

$$
\begin{array}{ll}
y(0)=0 & y\left(T_{\text {final }}\right)=1 \\
\dot{y}(0)=0 & \dot{y}\left(T_{\text {final }}\right)=0 \\
\ddot{y}(0)=0 & \ddot{y}\left(T_{\text {final }}\right)=0 .
\end{array}
$$

\section{Parameterization of Jerk Profiles}

The optimal jerk-constrained control profile will be the combination of a number of time-delayed ramp functions with different slopes. The requirement of time-optimality can be satisfied by ensuring that $u(t)$ is in saturation for as much time as possible during the maneuver. This is justified by the bang-bang principle [10], which states that if an optimal control exists, then there is always a bang-bang control profile that is optimal. Hence, if the optimal control is unique, it is bang-bang. For the controller presented in this paper, the jerk is the control variable. Thus, the jerk profile will be bang-bang, resulting in a ramping control input. For large permissible amounts of jerk, the control will reach the saturation level. Then a bang-off-bang profile will be used. In the off-phases, the control input $u(t)$ remains saturated, exerting the maximum possible control authority.

It will be assumed that the actuator has symmetric limits in $u(t)$, therefore, $u_{\max }=-u_{\min }=1$. The time rate of change is limited by $|\dot{u}(t)| \leqslant J$.

The control profile is realized by a time-delay filter which drives an integrator as shown in Fig. 1. The transfer function of this filter is given as

$$
G(s)=\frac{J}{s} \sum_{i=1}^{n} A_{i} e^{-s T_{i}}
$$

with

$$
A_{i} \in\{-2,-1,1,2\} \forall i .
$$

For the first switch, i.e., $i=1, A_{1}=1$, and $T_{1}=0$. Similarly, for the last switch, i.e., $i=n, A_{n} \in\{-1,1\}$, and $T_{n}=T_{\text {final }}$.

3.1 Structure of Jerk Profiles for Undamped Systems. The constraints for the time optimal control problem are derived using the technique proposed by Singh and Vadali [7], which re- 
quires cancellation of the poles of the system by the zeros of the time delay filter. The benchmark system will be the Floating Oscillator as shown in Fig. 2. For this system, augmented by an integrator, there are two undamped poles and three poles at the origin of the complex plane.

A general result which is exploited in this work is the fact that a transfer function has at least $n$ zeros at $s_{0}$, if the function evaluated at $s_{0}$ and its first $n-1$ derivatives with respect to $s$, evaluated at $s_{0}$, are zero. This can be stated as

$$
G(s)=\left.\left(s-s_{0}\right)^{n} R(s) \leftrightarrow G(s)\right|_{s=s_{0}}=0
$$

and

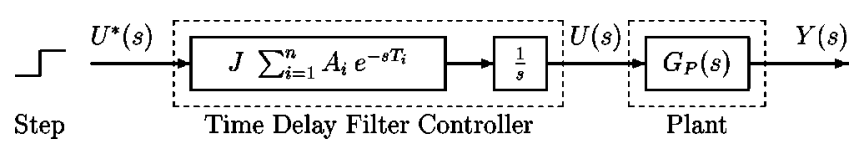

Fig. 1 Time delay filter structure

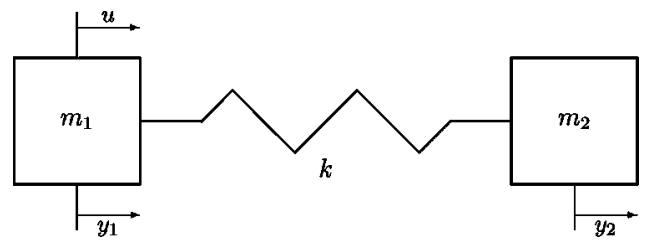

Fig. 2 Floating oscillator
Infinite Jerk Profile

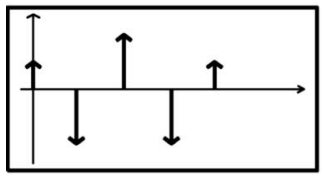

Uncollapsed Jerk Profile

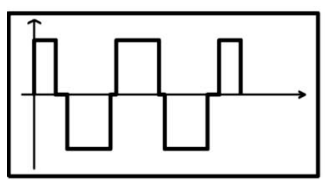

Partially Collapsed Jerk Profile

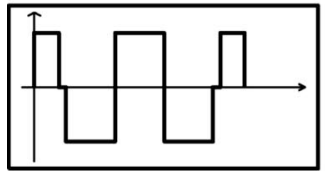

Intermediate Jerk Profile

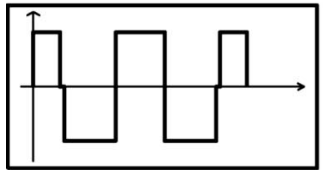

$$
\left.\frac{\partial^{i} G(s)}{\partial s^{i}}\right|_{s=s_{0}}=0 \forall i=1 \ldots(n-1) .
$$

In order to cancel three poles at the origin, the transfer function and its first two partial derivatives with respect to $s$ evaluated at $s=0$ must all be zero. It can be shown that all of these constraints are satisfied for a control profile which is point-symmetric about the mid-maneuver time. Thus, only those control profiles are considered in the following. By constraining the admissible set of control profiles to those point-symmetric profiles, the number of design variables describing the control profile's shape can practically be halved. In addition to the three poles at the origin of the $s$-plane, care must also be taken of the oscillatory pair of poles. Zeros of the time-delay filter will be used to cancel this pair of poles. For control profiles that are point-symmetric to the midmaneuver time, the two pole cancellation constraints, $\mathfrak{R}\{G(j \omega)\}$ $=0$ and $\Im\{G(j \omega)\}=0$, result in the same constraint equation for any arbitrary $s= \pm j \omega, \omega \in(0+, \infty)$.

Finally, a constraint must be imposed that governs the dynamics of the rigid body mode. It results in the equation

$$
y_{r}\left(T_{\text {mid }}\right)=\int_{0}^{T_{\text {mid }}}\left(\int_{0}^{\tau_{2}} \frac{u\left(\tau_{1}\right)}{m_{r}} d \tau_{1}\right) d \tau_{2}=\frac{1}{2}
$$

for a final desired displacement of unity. $m_{r}$ refers to the mass associated with the rigid body mode.

\section{Variation of Jerk Profiles}

In this section, the entire spectrum of possible jerk profiles is described. The structure of the control profiles starting from $J$ $\rightarrow \infty$ to $J \rightarrow 0$ is studied. For each profile, a short explanation will
$\mathrm{J} \rightarrow \infty \quad$ Transition Jerk Profile

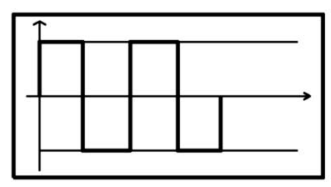

$\mathrm{J}=2$

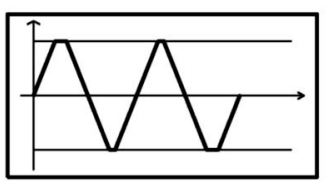

$\mathrm{J}=1.754$

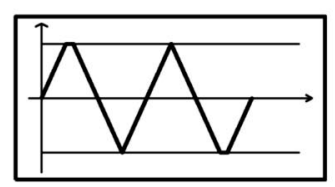

$\mathrm{J}=1.6$

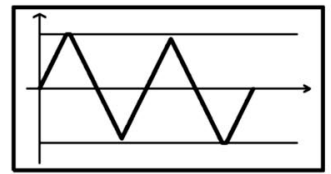

Triangular Jerk Profile

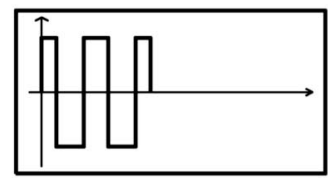

Totally Collapsed Jerk Profile
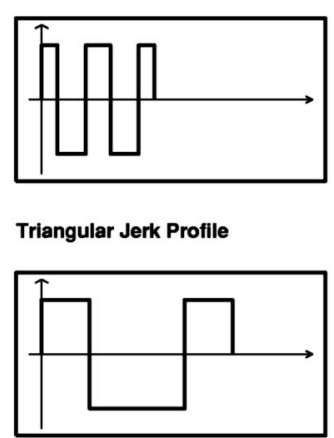

Very Small Jerk Profile

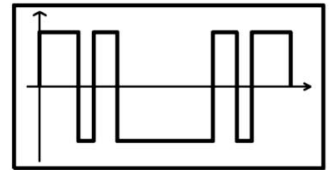

Right Profiles: $x$-axis: $0<t<12, y$-axis scaled to fit The left diagram shows the jerk, the right diagram the control input $u$. The thin line denotes the limits $u=1$ and $u=-1$.
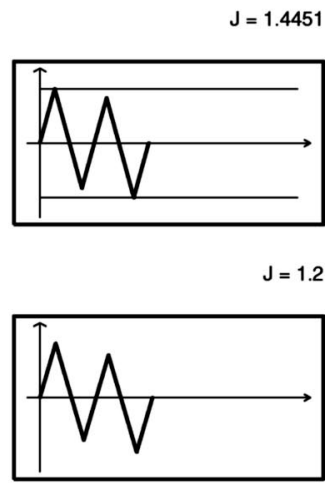

$\mathrm{J}=0.0912$

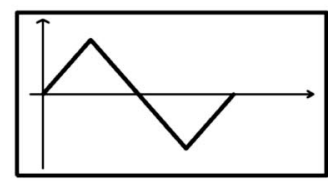

$\mathrm{J}=0.05$

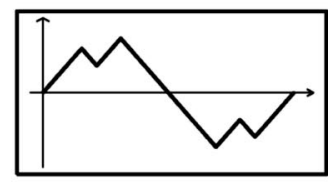

Fig. 3 Jerk profiles 
Table 1 Coefficients for the control sequences

\begin{tabular}{|c|c|c|c|c|c|c|c|c|c|}
\hline$\overline{\mathbf{J}}$ & 2 & 1.7540 & 1.6 & 1.4451 & 1.2 & 0.3 & 0.0912 & 0.05 & 0.02 \\
\hline $\begin{array}{l}\mathbf{A}_{1} \\
\mathbf{T}_{1} \\
\mathbf{A}_{2} \\
\mathbf{T}_{2} \\
\mathbf{A}_{3}\end{array}$ & $\begin{array}{r}2.0000 \\
0.0000 \\
-2.0000 \\
0.5000 \\
-2.0000\end{array}$ & $\begin{array}{r}1.7540 \\
0.0000 \\
-1.7540 \\
0.5701 \\
-1.7540\end{array}$ & $\begin{array}{r}1.6000 \\
0.0000 \\
-1.6000 \\
0.6250 \\
-1.6000\end{array}$ & $\begin{array}{r}1.4451 \\
0.0000 \\
-2.8901 \\
0.6920 \\
2.8901\end{array}$ & $\begin{array}{r}1.2000 \\
0.0000 \\
-2.4000 \\
0.7366 \\
2.4000\end{array}$ & $\begin{array}{r}0.3000 \\
0.0000 \\
-0.6000 \\
1.2604 \\
0.6000\end{array}$ & $\begin{array}{r}0.0912 \\
0.0000 \\
-0.1824 \\
2.2214 \\
0.1824\end{array}$ & $\begin{array}{r}0.0500 \\
0.0000 \\
-0.1000 \\
1.8046 \\
0.1000\end{array}$ & $\begin{array}{r}0.0200 \\
0.0000 \\
-0.0400 \\
3.4994 \\
0.0400\end{array}$ \\
\hline $\begin{array}{l}\mathbf{T}_{3} \\
\mathbf{A}_{4} \\
\mathbf{T}_{4} \\
\mathbf{A}_{5} \\
\mathbf{T}_{5}\end{array}$ & $\begin{array}{l}0.7687 \\
2.0000 \\
1.7687 \\
2.0000 \\
1.9008\end{array}$ & $\begin{array}{r}0.7384 \\
3.5080 \\
1.8787 \\
-3.5080 \\
3.0189\end{array}$ & $\begin{array}{r}0.7162 \\
3.2000 \\
1.9140 \\
-3.2000 \\
3.0596\end{array}$ & $\begin{array}{r}1.9584 \\
-2.8901 \\
3.1071 \\
2.8901 \\
4.3735\end{array}$ & $\begin{array}{r}2.0475 \\
-2.4000 \\
3.1963 \\
2.4000 \\
4.5073\end{array}$ & $\begin{array}{r}2.9679 \\
-0.6000 \\
3.8621 \\
0.6000 \\
5.5696\end{array}$ & $\begin{array}{r}6.6643 \\
-0.0912 \\
8.8858\end{array}$ & $\begin{array}{r}2.5064 \\
-0.1000 \\
3.6217 \\
0.1000 \\
8.0579\end{array}$ & $\begin{array}{r}4.5117 \\
-0.0400 \\
4.7325 \\
0.0400 \\
10.1482\end{array}$ \\
\hline $\begin{array}{l}\mathbf{A}_{6} \\
\mathbf{T}_{6} \\
\mathbf{A}_{7} \\
\mathbf{T}_{7} \\
\mathbf{A}_{8}\end{array}$ & $\begin{array}{r}-2.0000 \\
2.9008 \\
-2.0000 \\
3.0330 \\
2.0000\end{array}$ & $\begin{array}{r}1.7540 \\
4.1592 \\
1.7540 \\
4.3274 \\
-1.7540\end{array}$ & $\begin{array}{r}1.6000 \\
4.2575 \\
1.6000 \\
4.3486 \\
-1.6000\end{array}$ & $\begin{array}{r}-1.4451 \\
5.0655\end{array}$ & $\begin{array}{r}-1.2000 \\
5.2438\end{array}$ & $\begin{array}{r}-0.3000 \\
6.8299\end{array}$ & & $\begin{array}{r}-0.1000 \\
9.1732 \\
0.1000 \\
9.8750 \\
-0.0500\end{array}$ & $\begin{array}{r}-0.0400 \\
10.3690 \\
0.0400 \\
11.3813 \\
-0.0200\end{array}$ \\
\hline $\begin{array}{l}\mathbf{T}_{8} \\
\mathbf{A}_{9} \\
\mathbf{T}_{9} \\
\mathbf{A}_{10} \\
\mathbf{T}_{10}\end{array}$ & $\begin{array}{r}4.0330 \\
2.0000 \\
4.3017 \\
-2.0000 \\
4.8017\end{array}$ & 4.8976 & 4.9736 & & & & & 11.6796 & 14.8807 \\
\hline
\end{tabular}

also elucidate the reasons behind its name. The different profiles evolving during the increasing limitation of the jerk $J$ are illustrated in Fig. 3.

For $J \rightarrow \infty$, the control profile approaches the time optimal bang-bang-profile reported, e.g., by Singh and Vadali [9], which has been termed the Infinite Jerk Profile. Next comes the Uncollapsed Jerk Profile. Here, the control $u(t)$ will go to its upper and lower limits alternately. As will be seen in the following, some of the switches will coincide upon limiting the permissible amount of jerk. This is termed Collapse of Switches. Upon reducing the maximum allowable amount of jerk $J$, this collapse of switches will happen, thus the name Partially Collapsed Jerk Profile. The next resulting profile is the Intermediate Jerk Profile, for which the switches are not far enough apart for $u(t)$ to get saturated in each interval. For the Transition Control Profile, another collapse of switches takes place. Here, the resulting control profile $u(t)$ will touch its limits $|u(t)|=1$ only at two discrete points during the entire maneuver. Since this is the first non-saturating control profile, time optimality can be shown using Pontryagin's principle. This technique has been shown in the literature $[8,9]$ for infinite jerk profiles.

The next profile, which will emerge, is the Totally Collapsed Jerk Profile. At this point, all pairs of switches have collapsed, so that $|u(t)|<1$. The next distinct jerk profile is the Triangular Profile which gets its name from the triangular shape of the resulting control profile. From here on, new switches have to be introduced into the jerk profile which finally leads to the Very Small Jerk Profile.

The jerk profiles starting from the Transition Jerk Profile result in control profiles which never reach their limits in $u(t)$. Therefore, one can exploit Pontryagin's principle to prove optimality of the resulting control profile.

The above development has been carried out for undamped flexible structures. The proposed technique can be extended to damped structures by eliminating the requirement that the jerk profile has to be symmetric about the mid-maneuver time.

\section{Numerical Example}

The existence of all jerk profile structures is illustrated on the benchmark Floating Oscillator problem (Fig. 2) with $m_{1}=1, m_{2}$ $=1$ and $k=1$. Numerical values of the parameters of the optimal control profile are tabulated in Table 1 . The control profile is given as

Journal of Dynamic Systems, Measurement, and Control

$$
u(t)=\sum_{i} A_{i}\left\langle t-T_{i}\right\rangle,
$$

with $\langle x\rangle=x \mathcal{H}(x)$ and $\mathcal{H}(x)$ denotes the Heaviside function.

The effect of varying the jerk constraint on the switching times of the optimal jerk profile for a maneuver of unit length is studied. Figure 4 illustrates the change in the structure of the optimal jerk profile and the variation of the magnitudes of the switching times. In the diagram, the thick vertical lines denote borders, i.e., discrete values of jerk where the control profile changes its overall shape. The boxes show the shape of the control profile in different intervals. The thin arrows correlate the switches of the control profiles with the switching time trajectories.

It is clear from the figure that as $J \rightarrow \infty$, the optimal control profile tends to the unconstrained time-optimal solution derived by Wie and Liu [8] and Singh and Vadali [7]. The inner pairs of switches collapse for a jerk of $J=1.754$, followed by the outer pairs, which collapse for a jerk of $J=1.4451$. Finally, for the transition from the Totally Collapsed Jerk Profile to the Very Small Jerk Profile, the middle pair of switches collapses for a jerk of $J=0.0912$, resulting in the Triangular Profile. For a jerk less than $J=0.0912$, two new pairs of switches are introduced into the optimal jerk profile. This figure also shows the relation between the maximum allowable jerk and the final time, which is represented by the topmost line. Reducing the jerk from $J \rightarrow \infty$ to $J$ $=2$, for example, increases the final time from 4.2179 to 4.8017 .

Figure 5 shows a typical control profile along with the system response.

\section{Conclusions}

In this paper, a technique to design time-optimal jerk limited control sequences is presented. The controller consists of a time delay filter with a concatenated integrator. The integrator is included to account for the finite limits on the jerk. For undamped systems, the control profile is symmetric about its midpoint. Considering this property, the number of design variables can be reduced drastically. The design method can also be extended to damped systems by annulling these symmetry requirement.

The proposed technique has been illustrated on the benchmark Floating Oscillator problem. Upon introducing limits on the maximum allowable jerk, a number of different control profiles can evolve, which range from a three switch to a six switch profile. For specific values of jerk and maneuvers, a triangular control 


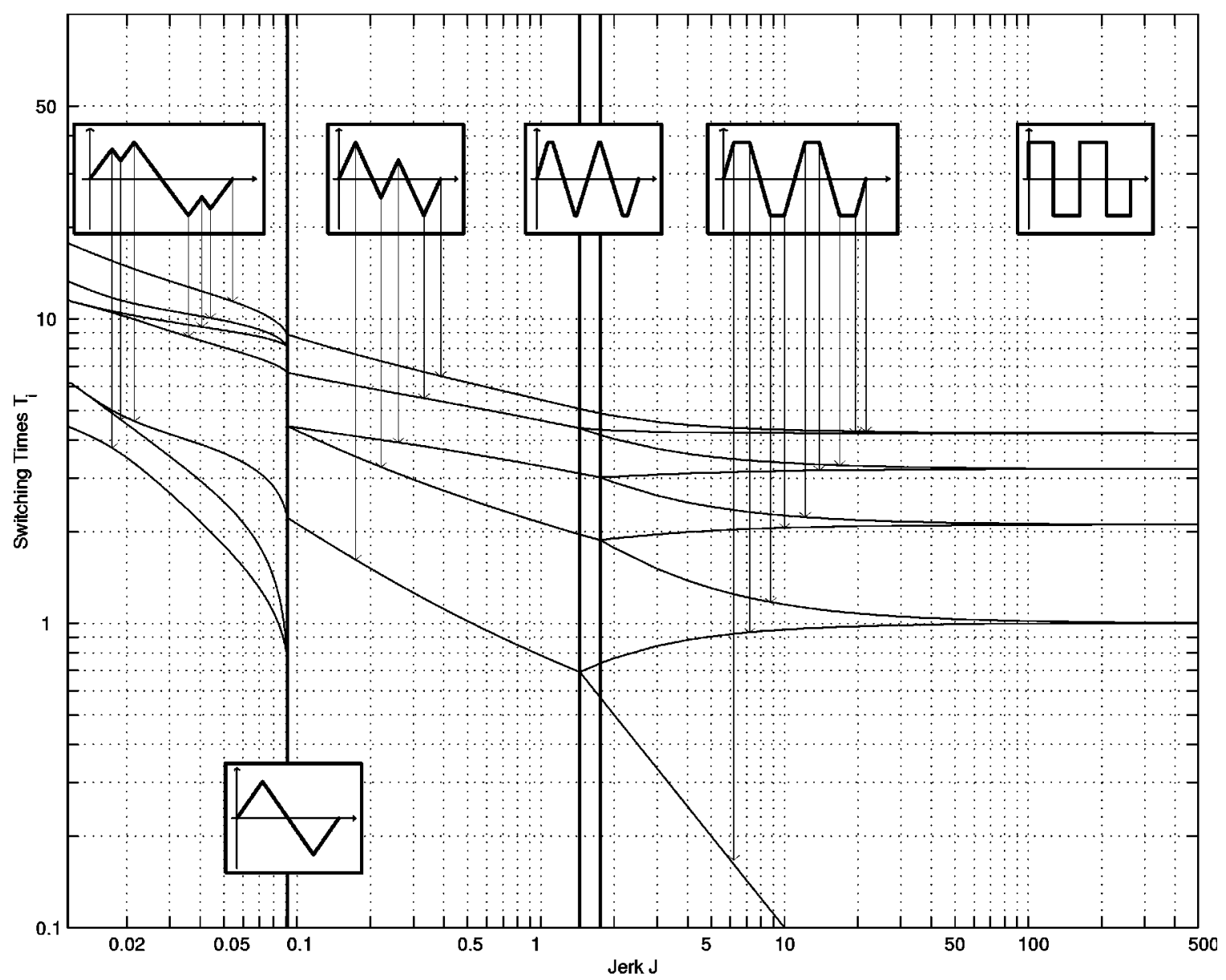

Fig. 4 Trajectories of the switching times $(J \in[0.11,500])$
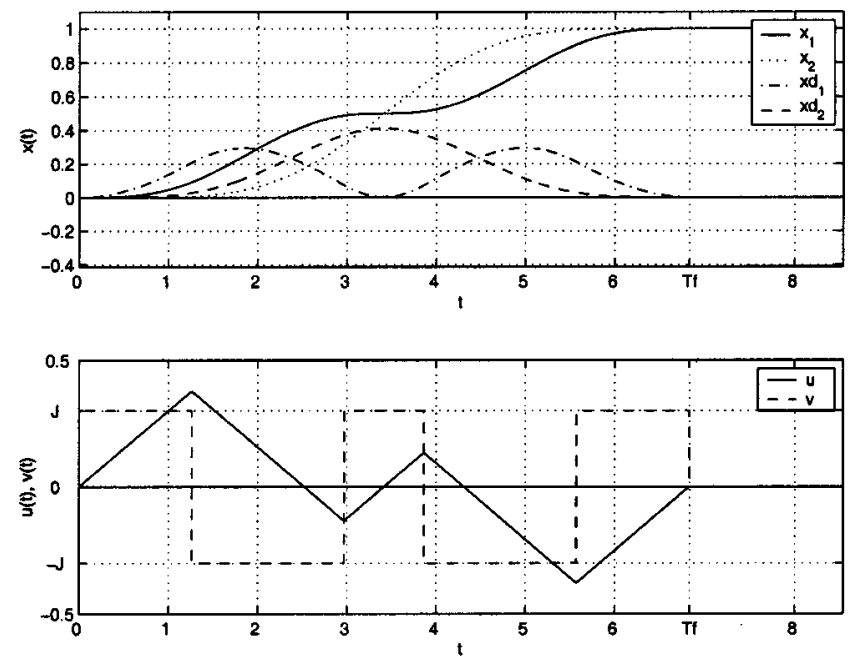

Fig. 5 Control profile and system response for $J=0.3$

profile is optimal. The variation of the switching profiles as a function of jerk has been presented in detail. It has been shown how the entire range of jerk can be divided into different regions by the shape of the control profile. Figures have been included that clearly illustrate the development of the switching times as a function of the maximum permissible jerk.
Numerical results show that accepting a small increase in the final time, the jerk can be reduced considerably. The restriction in the maximum time rate of change of the control inputs also leads to a roll-off of the high frequency spectrum of the control profile.

\section{References}

[1] Junkins, J. L., Rahman, Z., and Bang, H., 1990, "Near-Minimum Time Maneuvers of Flexible Vehicles: A Liapunov Control Law Design Method," Mechanics and Control of Large Flexible Structures, AIAA Publication, Washington, DC.

[2] Ballhaus, W. L., Rock, S. M., and Bryson, A. E., 1992, "Optimal Control of a Two-Link Flexible Robotic Manipulator Using Time-Varying Controller Gains," Amer. Astronautics Soc. Paper No. 92-055.

[3] Bhat, S. P., and Miu, D. K., 1991, "Minimum Power and Minimum Jerk Control and its Application in Computer Disk Drives," IEEE Trans. Magn., 27(6), pp. 4471-4475.

[4] Singhose, W. E., Porter, L. J., and Seering, W. P., 1997, "Input Shaped Control of a Planar Gantry Crane with Hoisting," 1997 American Control Conf., Albuquerque, NM.

[5] Smith, O. J. M., 1957, "Posicast Control of Damped Oscillatory Systems," Proc. of IRE, pp. 1249-1255.

[6] Singer, N. C., and Seering, W. P., 1990, "Preshaping Command Inputs to Reduce System Vibrations," ASME J. Dyn. Syst., Meas., Control, 115, pp 76-82.

[7] Singh, T., and Vadali, S. R., 1993, "Robust Time-Delay Control," ASME J. Dyn. Syst., Meas., Control, 115(2A), pp. 303-306.

[8] Liu, Q., and Wie, B., 1992, "Robust Time-Optimal Control of Uncertain Flexible Spacecraft," J. Guidance Control Dyn., 15(3), pp. 597-604.

[9] Singh, T., and Vadali, S. R., 1994, "Robust Time-Optimal Control: Frequency Domain Approach," J. Guidance Control Dyn., 17(2), pp. 346-353.

[10] Hermes, H., and Lasalle, J. P., 1969, Functional Analysis and Time Optimal Control, Academic Press, New York, NY. 